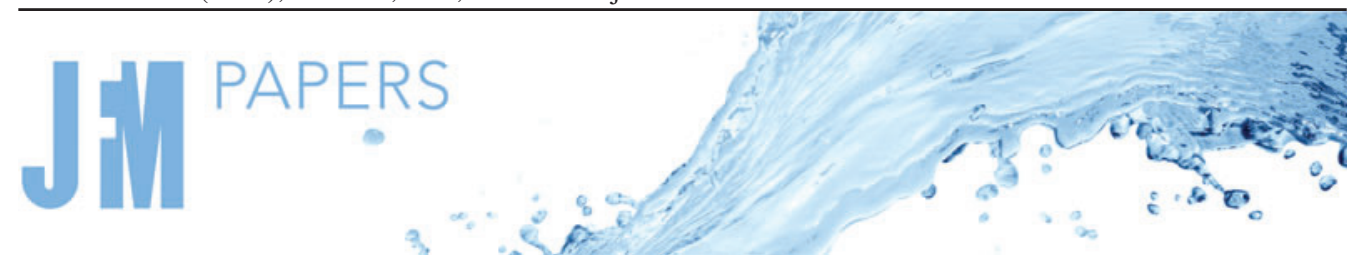

\title{
Numerical investigation of turbulence of surface gravity waves
}

\author{
Zhou Zhang ${ }^{1}$ and Yulin $\operatorname{Pan}^{1, \dagger}$ \\ ${ }^{1}$ Department of Naval Architecture and Marine Engineering, University of Michigan, Ann Arbor, \\ MI 48109, USA
}

(Received 2 August 2021; revised 18 October 2021; accepted 8 December 2021)

In this paper, we numerically study the wave turbulence of surface gravity waves in the framework of Euler equations of the free surface. The purpose is to understand the variation of the scaling of the spectra with wavenumber $k$ and energy flux $P$ at different nonlinearity levels under different forcing/free-decay conditions. For all conditions (free decay and narrow-band and broad-band forcing) that we consider, we find that the spectral forms approach the wave turbulence theory (WTT) solution $S_{\eta} \sim k^{-5 / 2}$ and $S_{\eta} \sim P^{1 / 3}$ at high nonlinearity levels. With a decrease of nonlinearity level, the spectra for all cases become steeper, with the narrow-band forcing case exhibiting the most rapid deviation from WTT. We investigate bound waves and the finite-size effect as possible mechanisms causing the spectral variations. Through a tri-coherence analysis, we find that the finite-size effect is present in all cases, which is responsible for the overall steepening of the spectra and the reduced capacity of energy flux at lower nonlinearity levels. The fraction of bound waves in the domain generally decreases with the decrease of nonlinearity level, except for the narrow-band case, which exhibits a transition at a critical nonlinearity level below which a rapid increase is observed. This increase serves as the main reason for the fastest deviation from WTT with the decrease of nonlinearity in the narrow-band forcing case.

Key words: surface gravity waves, homogeneous turbulence

\section{Introduction}

The normal state of the ocean surface is characterized by a large number of waves at difference scales subject to nonlinear interactions in the presence of wind forcing and viscous dissipation. In such a state, referred to as wave turbulence, a continuous surface

$\dagger$ Email address for correspondence: yulinpan@umich.edu

(C) The Author(s), 2022. Published by Cambridge University Press. This is an Open Access article, distributed under the terms of the Creative Commons Attribution licence (https://creativecommons. org/licenses/by/4.0/), which permits unrestricted re-use, distribution, and reproduction in any medium, provided the original work is properly cited. 


\section{Z. Zhang and Y. Pan}

elevation spectrum is usually developed with a power-law form in the inertial range, as a result of the energy cascade through scales. In the framework of weak turbulence theory (WTT), the wave spectra can be analytically computed based on the assumptions of weak nonlinearity, infinite domain and phase stochasticity, leading to the so-called Kolmogorov-Zakharov spectra (Zakharov \& Filonenko 1967). For surface gravity waves, the omnidirectional Kolmogorov-Zakharov wavenumber spectra have the form

$$
S_{\eta}(k) \propto P^{1 / 3} k^{-5 / 2},
$$

where $P$ is the energy flux to small scales and $k$ is the wavenumber.

Since the assumptions in WTT are difficult to satisfy in finite facilities, experimental attempts to verify WTT often show deviations from (1.1). In terms of the scaling $S_{\eta}(k) \sim$ $k^{\alpha}$ (or its frequency counterpart), different values of $\alpha$ are observed in experiments under different conditions (e.g. forcing with different amplitudes and bandwidths), and these findings are sometimes in disagreement with one another. For example, observing waves generated by a localized wave maker, Falcon, Laroche \& Fauve (2007), Nazarenko et al. (2010), Deike et al. (2015) and Denissenko, Lukaschuk \& Nazarenko (2007) show that the spectral slope $\alpha$ depends on the forcing condition and approaches (1.1) at high (or certain) forcing amplitudes. In contrast, insensitivity of $\alpha$ to the forcing amplitude is reported in Issenmann \& Falcon (2013), Aubourg \& Mordant (2016) and Herbert, Mordant \& Falcon (2010), with the former two experiments forced by horizontal vibrations of the whole container and the third one by a wave maker. The spectra obtained in these three experiments are all steeper than (1.1) and inconsistent with each other. Issenmann \& Falcon (2013) further suggest that their forcing by vibration provides a more homogeneous and isotropic spectrum than the forcing by a wave maker, and therefore is potentially more consistent with WTT. In addition, Cobelli et al. (2011) show that the observed spectra also depend strongly on the bandwidth of the forcing provided by a wave maker.

The situation for the scaling between $S_{\eta}(k)$ and $P$ is more elusive, with Falcon et al. (2007) and Issenmann \& Falcon (2013) reporting a scaling $S_{\eta} \sim P$ in disagreement with (1.1). However, their measurement of $P$ is based on the mean power injected by a wave maker, which may lead to inconsistency with the concept of energy flux due to the broad-scale dissipation (Deike, Berhanu \& Falcon 2014; Pan \& Yue 2015). More specifically, the dissipation occurring at all scales may result in significant dissipation in the inertial range (or even at larger scales), potentially rendering the energy input rate to be dominated by the large-scale dissipation instead of the energy flux across the inertial range. Using the same measurement of $P$, Cobelli et al. (2011) further suggest that the $P$ and $P^{1 / 3}$ scaling can be realized with respectively broad-band and narrow-band forcing.

The inconsistencies in experiments (with WTT and with one another) are usually attributed to factors including the finite-size effect, bound waves and coherent structures. First, the finite-size effect (e.g. Pushkarev \& Zakharov 2000; Lvov, Nazarenko \& Pokorni 2006; Nazarenko 2006) occurs at low nonlinearity level when the nonlinear broadening is not sufficient to overcome the discreteness of $k$ caused by the finite size of the facility. This is in contrast to the continuous $k$ configuration in deriving (1.1). Second, bound waves can be considered as wave components not satisfying the dispersion relation, generated from harmonics (i.e. non-resonant interactions) or distortion of the carrier wave (Plant et al. 1999, 2004; Herbert et al. 2010). It is found in Michel et al. (2018) and Campagne et al. (2019) that bound waves are dominant at high frequencies and are likely responsible for the deviation of the measurements from WTT (and its dependence on forcing amplitudes). Third, coherent structures (such as rogue waves and wave breaking) occurring at high nonlinearity levels are not described by WTT, and thus may lead to spectra different 
from (1.1). Candidate theories to model such spectra include the Phillips spectra (Phillips 1958) and the Kuznetsov spectra (Kuznetsov 2004). Finally, all experiments involve other complexities such as reflection from boundaries, broad-scale dissipation and the surface tension effect which inevitably affect the spectra to some extent.

In numerical simulations, we are able to have a better control of the wave field by precisely specifying the forcing/dissipation and the implementation of periodic boundary conditions. This offers us a clean environment to study wave turbulence at various conditions free of the complexities that are present in experiments. Existing work includes Dyachenko, Korotkevich \& Zakharov (2004) and Lvov et al. (2006) for forcing turbulence and Onorato et al. (2002) and Yokoyama (2004) for free-decay turbulence of gravity waves in the context of Euler equations. While all these works report a scaling $S_{\eta}(k) \sim k^{-5 / 2}$ consistent with (1.1), the simulation (in each of them) is conducted at a single nonlinearity level, and therefore is not capable of resolving/understanding the sensitivity of the spectra to various conditions and their scaling with $P$. On the other hand, free-decay turbulence is studied for capillary waves in Pan \& Yue (2014), which reveals steepened spectra with a decrease of nonlinearity level. However, the finding cannot be naively applied to gravity waves, because the spectral behaviour at low nonlinearity critically depends on the discrete resonant manifold (Hrabski \& Pan 2020) which has not been characterized for surface gravity waves.

In this work, we conduct a numerical study of the spectral properties of gravity wave turbulence at different forcing (in terms of bandwidths and amplitudes) and free-decay (with relatively broad-band initial data) conditions. The purpose is to elucidate the mechanisms underlying the spectral variation under different conditions. In particular, we focus on the hypothetical mechanisms of finite-size effect and bound waves, and leave the study of coherent structures to our future work which directly simulates the two-phase Navier-Stokes equations (since only some of the coherent structures can be simulated in the framework of Euler equations). We also envision the presented numerical findings to be eventually used to explain the aforementioned experimental observations with the necessary considerations of further complexities in experiments.

The outline and some main findings of the paper are as follows. In $\S 2$, we present the numerical set-up of the Euler equations under forcing and free-decay conditions. In $\S 3$, we show the numerical results including the scaling of the wave spectra with $k$ and $P$ at different nonlinearity levels. It is found that the WTT solution is approached at high nonlinearity levels for all conditions (free decay and narrow-band and broad-band forcing). The spectra deviate from WTT as the nonlinearity level decreases with the largest deviation rate observed in the narrow-band forcing case. Mechanisms leading to the variation of spectra with nonlinearity levels are discussed in terms of bound waves and the finite-size effect. Through a tri-coherence analysis we find that the finite-size effect is present at low nonlinearities for all cases, responsible for the overall steepening of the spectra and the reduced energy flux capacity. The fraction of bound waves generally decreases with a decrease of nonlinearity, but exhibits a sharp transition and explains the rapid deviation of the spectra from WTT in the narrow-band forcing case. The conclusions are provided in $\S 4$.

\section{Mathematical formulation}

We consider gravity waves on a two-dimensional free surface of an incompressible, inviscid and irrotational fluid. The flow can be described by a velocity potential $\phi(x, z, t)$ satisfying Laplace's equation. Here $\boldsymbol{x}=(x, y)$ is the horizontal coordinates, $z$ is the 


\section{Z. Zhang and Y. Pan}

vertical coordinate and $t$ is time. The surface velocity potential is defined as $\phi^{S}(\boldsymbol{x}, t)=$ $\left.\phi(x, z, t)\right|_{z=\eta}$, where $\eta(x, t)$ is the surface elevation. The evolutions of $\eta$ and $\phi^{S}$ satisfy the Euler equations in Zakharov form (Zakharov 1968):

$$
\begin{gathered}
\eta_{t}+\nabla_{x} \eta \cdot \nabla_{x} \phi^{S}-\left(1+\nabla_{x} \eta \cdot \nabla_{x} \eta\right) \phi_{z}=0, \\
\phi_{t}^{S}+\eta+\frac{1}{2} \nabla_{x} \phi^{S} \cdot \nabla_{x} \phi^{S}-\frac{1}{2}\left(1+\nabla_{x} \eta \cdot \nabla_{x} \eta\right) \phi_{z}^{2}=0,
\end{gathered}
$$

where $\phi_{z}(\boldsymbol{x}, t)=\partial \phi /\left.\partial z\right|_{z=\eta}$ is the surface vertical velocity and $\boldsymbol{\nabla}_{\boldsymbol{x}}=(\partial / \partial x, \partial / \partial y)$ denotes the horizontal gradient. In (2.2), we assume that the mass and time units are properly chosen such that density and gravitational acceleration both take values of unity (Dommermuth \& Yue 1987).

To integrate (2.1) and (2.2) in time, we use the higher-order spectral (HOS) method (Dommermuth \& Yue 1987; West et al. 1987). We use a nonlinearity order $M=3$ which includes nonlinear terms up to the third order allowing both three-wave and four-wave interactions. While the three-wave interactions are responsible for the generation of bound waves, the four-wave resonant interactions are considered the dominant energy transfer processes for gravity waves (e.g. Hammack \& Henderson 1993; Mei, Stiassnie \& Yue 2005). All simulations are conducted in a doubly periodic square domain of size $2 \pi \times 2 \pi$ corresponding to a fundamental wavenumber $k_{0}=1$, with a spatial resolution of $512 \times$ 512 (which is sufficient to capture the phenomena of interest, and chosen in consideration of the total computational cost of 25 simulations needed in this work). To account for the dissipation at high wavenumbers, we add two artificial terms respectively on the right-hand sides of (2.1) and (2.2):

$$
\begin{gathered}
D_{\eta}(k)=\gamma_{k} \eta, \\
D_{\phi^{S}}(k)=\gamma_{k} \phi^{S},
\end{gathered}
$$

with the dissipation coefficient $\gamma_{k}$ defined as

$$
\gamma_{k}=\gamma_{0}\left(k / k_{d}\right)^{v}
$$

where $\gamma_{0}, k_{d}$ and $v$ are parameters characterizing the dissipation. This formulation is equivalent to the low-pass filter operation used in Xiao et al. (2013), developed through a phenomenological matching with the measurement of dissipation in experiments. In the current work, we use values of $\gamma_{0}=-50, k_{d}=115$ and $v=30$. We note that this formulation provides a sharp transition to the dissipation range above $k \approx k_{d}$, which is essential for us to use the dissipation rate to measure the energy flux in our numerical studies (i.e. free of the broad-scale dissipation effect).

In this work, we conduct simulations for a free-decay case, and two cases with external forcing of broad and narrow bandwidth. For the free-decay case, we use as initial condition a wavenumber spectrum converted from a directional frequency spectrum $S_{D}(\omega, \theta)=$ $D(\theta) G(\omega)$, where $\theta$ is the directional angle with respect to the positive $x$ direction. The spreading function $D(\theta)$ characterizes the angular dependence of the spectra and is chosen to be a cosine-squared function:

$$
D(\theta)= \begin{cases}\frac{2}{\pi} \cos ^{2} \theta, & |\theta| \leq \pi / 2, \\ 0, & |\theta|>\pi / 2 .\end{cases}
$$

Instead of using an isotropic initial condition, we use here an angle-dependence spectrum to better represent the real ocean condition (Tanaka 2001; Onorato et al. 2002). 
To further justify this choice, we have also verified that using different spreading angles in (2.6) does not critically affect the results presented in this paper.

The frequency spectrum $G(\omega)$ is set to the form of a Gaussian function:

$$
G(\omega)=\frac{B}{\sqrt{2 \pi \sigma^{2}}} \exp \left[-\frac{\left(\omega-\omega_{p}\right)^{2}}{2 \sigma^{2}}\right],
$$

with $\sigma=0.4$ and $B$ a parameter characterizing the initial effective steepness $\epsilon$ as a measure of the nonlinearity level, which is defined by

$$
\epsilon=k_{p} H_{s} / 2
$$

where $H_{S}$ is the significant wave height and $k_{p}$ the peak wavenumber. We use $\omega_{p}=\sqrt{10}$ corresponding to $k_{p}=10$ in the initial wavenumber spectrum.

For the forcing cases, the initial condition is a quiescent water surface and the waves are generated by forcing with different bandwidths and amplitudes. Numerically the forcing is modelled by an artificial pressure term $Q(\theta, k, t)=H(\theta) F(k, t)$ added to the right-hand side of (2.2). The angular cut-off function $H(\theta)$ takes a value of one for $|\theta| \leq \pi / 4$ and zero otherwise. We use a relatively narrow forcing spreading angle here because the HOS method is less numerically stable for cases with broader-angle and isotropic forcing of this type. While the reason for this requires further study, to obtain results for a broad range of nonlinearity as shown in $\S 3$, the usage of the current spreading angle is practically necessary. The function $F(k, t)$ takes the form (e.g. Dyachenko et al. 2004; Pan 2020)

$$
F(k, t)= \begin{cases}f_{k} \exp \left[-C t+\mathrm{i}\left(\omega_{k} t+R\right)\right], & t \leq T_{c}, \\ f_{k} \exp \left[-C T_{c}+\mathrm{i}\left(\omega_{k} t+R\right)\right], & t>T_{c},\end{cases}
$$

with

$$
f_{k}= \begin{cases}f_{0} \frac{\left(k-k_{1}\right)\left(k_{2}-k\right)}{\left(k_{1}-k_{2}\right)^{2}}, & k_{1} \leq k \leq k_{2} \\ 0, & \text { otherwise }\end{cases}
$$

where $f_{0}$ is the parameter determining the forcing amplitude, $\omega_{k}$ is the angular frequency for wavenumber $k$ calculated from the dispersion relation, $k_{1}$ and $k_{2}$ are the lower and upper bounds of the forcing range and $R$ is a random number uniformly distributed in $[0,2 \pi]$ that are different for each wave mode. In the broad-band and narrow-band cases, we use $\left[k_{1}, k_{2}\right]=[1,19]$ and $\left[k_{1}, k_{2}\right]=[9,11]$ respectively, both corresponding to a peak mode of $k_{p}=\left(k_{1}+k_{2}\right) / 2=10$. As described by (2.9), the forcing level decays exponentially in time with rate $C$ before $t=T_{c}$, and then remains constant. This provides a fast convergence to stationary turbulence state where the forcing balances the dissipation. We use values of $T_{c}=500 T_{p}$ and $C=\ln 5 / T_{c}$ (where $T_{p}$ is the peak period corresponding to $k_{p}$ ) which lead to favourable convergence rates in our study.

\section{Results}

In this section, we present the results from simulations of free-decay turbulence and forcing turbulence with broad and narrow bandwidths. The former is conducted at different effective steepness of the initial conditions, and the latter at different forcing amplitudes, in order to cover a sufficient range of nonlinearity levels. In the following, we focus on the scaling of $S_{\eta}(k)$ with $k$ and $P$ at different nonlinearity levels, and investigate the mechanisms underlying the variations. 


\section{Z. Zhang and Y. Pan}

\subsection{Spectral slopes}

We first define the omnidirectional wavenumber spectrum $S_{\eta}(k)$ (we neglect its $t$ dependence for simplicity in the definition) by

$$
S_{\eta}(k)=\int_{0}^{2 \pi}|\tilde{\eta}(\boldsymbol{k})|^{2} k \mathrm{~d} \theta
$$

where $\boldsymbol{k}=\left(k_{x}, k_{y}\right), k=|\boldsymbol{k}|$ and $\tilde{\eta}(\boldsymbol{k})$ is the spatial Fourier transform of $\eta(\boldsymbol{x})$ :

$$
\tilde{\eta}(\boldsymbol{k})=\iint_{[0,2 \pi] \times[0,2 \pi]} \eta(\boldsymbol{x}) \exp (-\mathrm{i} \boldsymbol{k} \cdot \boldsymbol{x}) \mathrm{d} \boldsymbol{x} .
$$

To demonstrate the (quasi-)stationarity of the spectral evolution, we define two integral measures $E_{\text {in }}$ and $\Psi_{\text {in }}$ respectively for the spectra and compensated spectra (which assigns more weight on the high-wavenumber part), given by

$$
\begin{gathered}
E_{\text {in }}(t) \equiv \int_{k_{c}}^{k_{d}} S_{\eta}(k, t) \mathrm{d} k, \\
\Psi_{\text {in }}(t) \equiv \int_{k_{c}}^{k_{d}} k^{5 / 2} S_{\eta}(k, t) \mathrm{d} k,
\end{gathered}
$$

where $k_{c}=19$ locates within the inertial range as is shown later (also corresponding to the upper bound of the broad-band forcing). We check the evolutions of $\Psi_{\text {in }} / E_{\text {in }}$ and $\Psi_{\text {in }}$ in free-decay and forcing cases to characterize their stationary state, where the denominator in the first quantity is used to account for the slow decay of energy level with time in the free-decay case.

The results obtained from free-decay cases are presented in figure 1. Figure 1(a) shows the evolution of $\Psi_{\text {in }} / E_{\text {in }}$ with different nonlinearity levels, measured by the value of $\epsilon$ evaluated at $t=500 T_{p}$. It can be seen that quasi-stationary states are established for all cases after $t=500 T_{p}$. A typical spectral evolution for the case with $\epsilon=0.151$ is also shown as an inset to demonstrate the convergence of the spectrum to a power-law state. The spectra at quasi-stationary states for different nonlinearity levels (i.e. values of $\epsilon$ ) are shown in figure $1(b)$. At high nonlinearity level of $\epsilon=0.151$, we observe a clear power-law spectrum which has a slope $\alpha \approx-5 / 2$ consistent with WTT solution (1.1) in an approximate range of $[10,65]$. We note that the power-law range ends at $k \approx 65$ which is smaller than $k_{d}=115$, similar to other numerical simulations with a sharp dissipation cutoff (e.g. Dyachenko et al. 2004; Pan \& Yue 2014), probably due to the interaction of the spectrum with the dissipation range that is not in a power-law form. With a decrease of nonlinearity level $\epsilon$, the power-law spectrum becomes shorter and steeper, reaching $\alpha \approx-3.4$ at $\epsilon=0.068$. This steepening of the spectra is in contrast to the results from Majda-McLaughlin-Tabak (MMT) turbulence with $\omega=k^{2}$ and quartet resonance (Hrabski \& Pan 2020), mainly because the latter forms a continuous resonant system (Faou, Germain \& Hani 2016) at low nonlinearity (we elaborate this more in $\S 3.4$ ). We also remark that $\epsilon=0.151$ is about the highest nonlinearity we can reach in the current HOS context. The simulation for this strongly nonlinear case is possible using the HOS method because of the damping terms (2.3) and (2.4) which phenomenologically account for the wave breaking (e.g. Xiao et al. 2013). Previous free-decay simulations of Onorato et al. (2002) and Yokoyama (2004) which result in $\alpha \approx-5 / 2$ are both conducted at a value of $\epsilon$ close to 0.15 (respectively 0.15 and 0.14 ).

The results from the forcing cases are shown in figure 2. The evolutions of $\Psi_{\text {in }}$ in the narrow-band and broad-band cases with different forcing amplitudes $f_{0}$ (resulting 

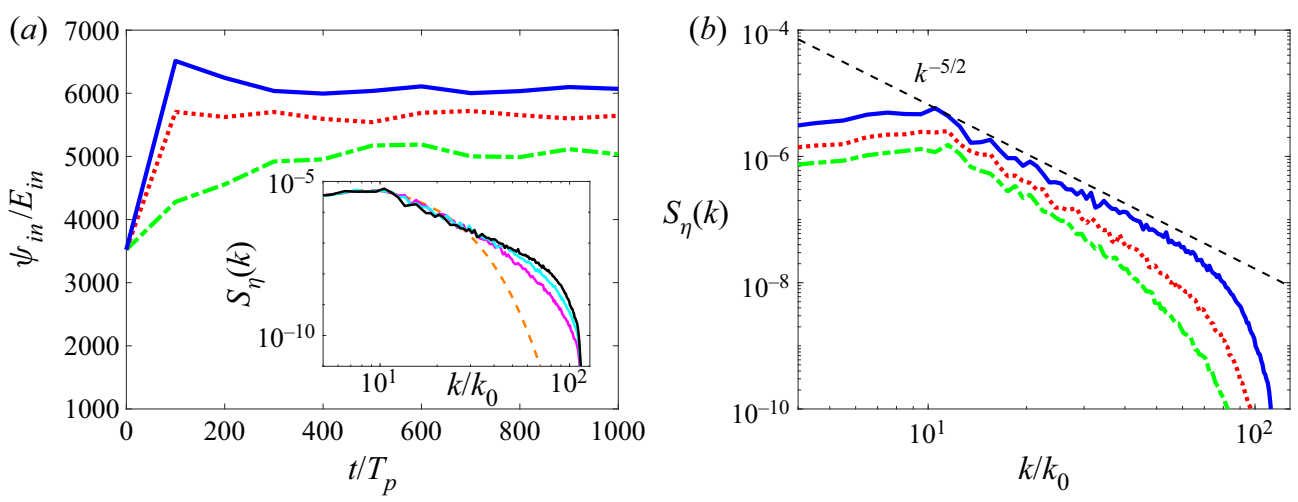

Figure 1. (a) Time evolution of $\Psi_{\text {in }} / E_{\text {in }}$ and (b) stationary spectra in free-decay cases with $\epsilon=0.068(-\cdots$, green), $0.106(\cdots$, red $)$ and $0.151(-$, blue $)$ evaluated at $t=500 T_{p}$ from different runs. Inset of $(a)$ shows wave spectra obtained at $t=0\left(---\right.$, orange), $100 T_{p}$ ( - , magenta), 200T $\left(-\right.$, cyan) and $500 T_{p}(-)$ with $\epsilon=0.151$. The theoretical power-law scaling $k^{-5 / 2}(---)$ is indicated in $(b)$ for reference.

in different values of $\epsilon$ at the stationary state) are plotted in figures $2(a)$ and $2(c)$, all showing stationary states in $\left[1000 T_{p}, 1500 T_{p}\right]$. The stationary power-law spectra obtained at $t=1500 T_{p}$ for the two cases are plotted in figures $2(b)$ and $2(d)$, respectively. For both cases, we observe that the spectral slopes $\alpha$ approach $-5 / 2$ at sufficiently high forcing/nonlinearity (consistent with previous work (Dyachenko et al. 2004)). The power-law ranges for both cases begin immediately above $k=10$, even though the forcing is applied to the range above $k=10$. This is an indication that the nonlinear interaction is strong enough (relative to the forcing) to dominate the dynamics in this range. With a decrease of nonlinearity, the spectra for both cases become steeper, which are also reported in experiments in Falcon et al. (2007), Nazarenko et al. (2010) and Deike et al. (2015). However, we observe that the spectra at low nonlinearity clearly show a dependence on the bandwidth of the forcing, with the one from the narrow-band case much steeper (even not showing a power law) than the one from the broad-band case. It can also be noticed that, for the narrow-band case, the spectrum at low nonlinearity level $(\epsilon=0.059)$ exhibits discrete superharmonic peaks, are explained through bound waves in $\S 3.3$.

To obtain a complete view of spectral slopes for both the free-decay and forcing cases, we plot the values of $\alpha$ in all cases as functions of the nonlinearity level in figure 3. This plot is limited above by the stability of the HOS method and below by the existence of a power-law spectrum. In practice, we consider a power-law spectrum not existing if the power-law range is less than 0.5 decade or if the spectrum is dominated by discrete peaks such as the one with low nonlinearity level in figure $2(d)$. We see that for all cases, the spectral slope $\alpha$ approaches the WTT value $-5 / 2$ at sufficiently high nonlinearity levels of $\epsilon \approx 0.15$. With a decrease of $\epsilon$, all spectra become steeper but with different steepening rates especially at relatively low nonlinearity level. It is also clear that the narrow-band forcing case shows a transition at $\epsilon_{c} \approx 0.11$, below which a very rapid steepening is observed. The mechanisms underlying these behaviours are further analysed in $\S \S 3.3$ and 3.4.

\subsection{Energy flux}

In this section, we investigate the scaling between the spectral level of $S_{\eta}(k)$ and the energy flux $P$. For the evaluation of $P$, we can directly compute the energy transfer 


\section{Z. Zhang and Y. Pan}
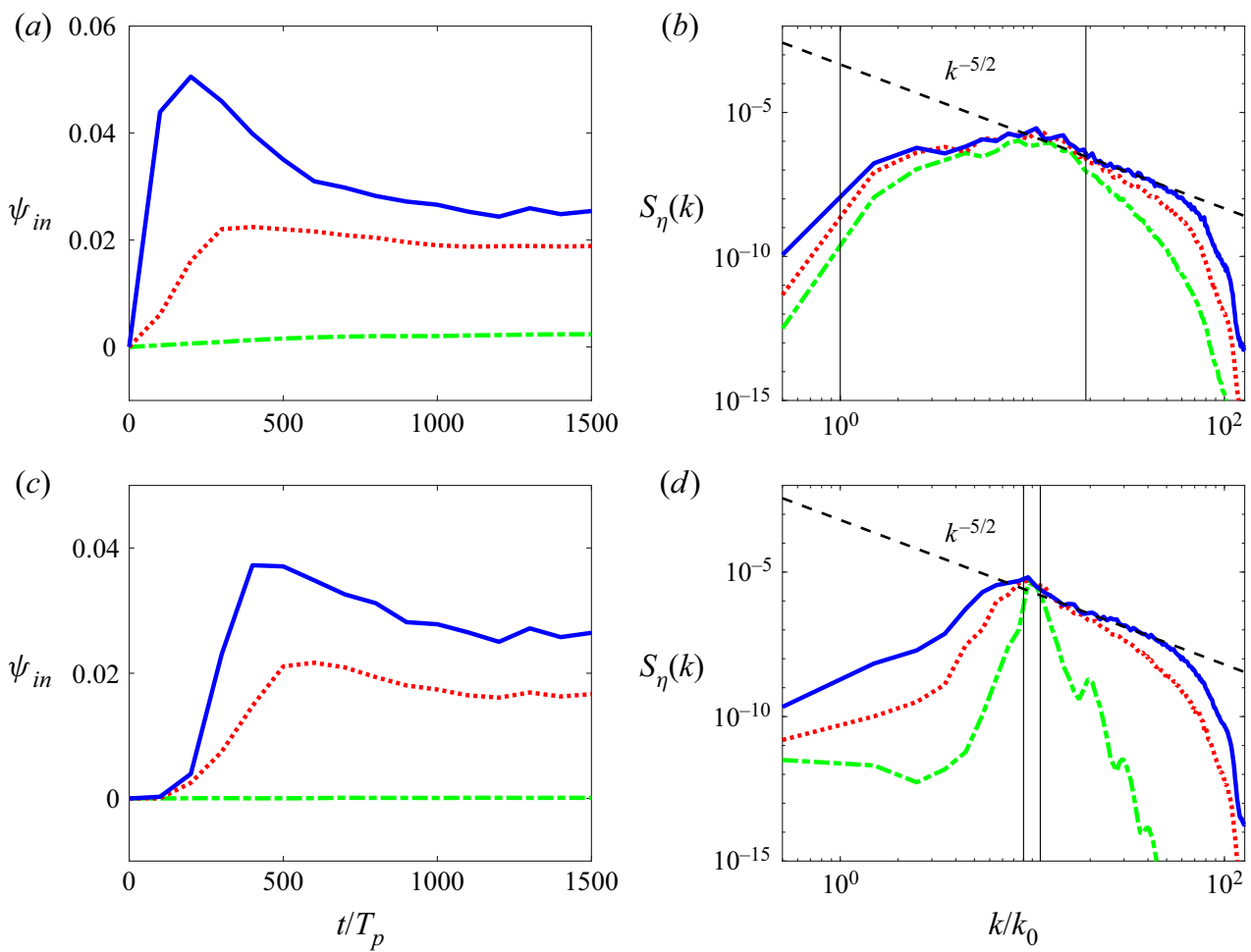

Figure 2. (a) Time evolution of $\Psi_{\text {in }}$ and $(b)$ stationary spectra obtained at $t=1500 T_{p}$ in the broad-band forcing case with $f_{0}=3 \times 10^{-7}, \epsilon=0.061(-\cdots$, green $) ; f_{0}=1.6 \times 10^{-6}, \epsilon=0.118(\cdot \cdots$, red $) ; f_{0}=3.2 \times 10^{-6}$, $\epsilon=0.145$ ( - , blue). (c) Time evolution of $\Psi_{\text {in }}$ and $(d)$ stationary spectra obtained at $t=1500 T_{p}$ in the narrow-band forcing case with $f_{0}=8 \times 10^{-7}, \epsilon=0.059\left(-\cdots\right.$, green); $f_{0}=4 \times 10^{-6}, \epsilon=0.114(\cdot \cdots$, red $)$; $f_{0}=1 \times 10^{-5}, \epsilon=0.148$ ( - , blue). The theoretical power-law scaling $k^{-5 / 2}(---)$ and boundaries of the forcing ranges $(-)$ are indicated in $(b, d)$ for reference.

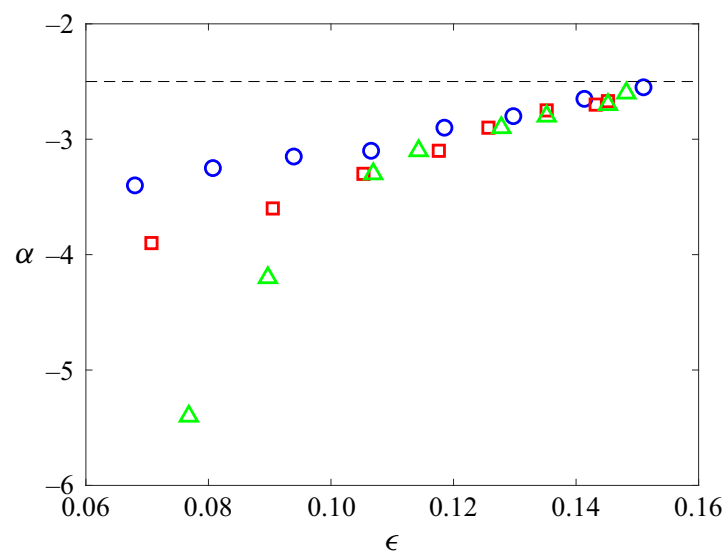

Figure 3. The spectral slope $\alpha$ as functions of effective steepness $\epsilon$ for the free-decay (o, blue), broad-band forcing $(\square$, red) and narrow-band forcing $(\triangle$, green) cases, compared with the prediction of WTT with $\alpha=$ $-5 / 2(---)$. 
due to nonlinear terms in the primitive equation (Hrabski \& Pan 2020) or use energy dissipation rate as a measure of $P$ (e.g. Pan \& Yue 2014). For dissipation localized at high wavenumbers (such as our cases), the two approaches are equivalent (as all energy flux through the inertial range is dissipated at high wavenumbers in a stationary state). Therefore, we use dissipation rate as a measure of $P$, which takes the form (Pushkarev \& Zakharov 1996; Pan \& Yue 2014)

$$
P=\iint_{\boldsymbol{k}} \gamma_{k}\left(|\tilde{\eta}(\boldsymbol{k})|^{2}+k\left|\widetilde{\phi}^{S}(\boldsymbol{k})\right|^{2}\right) \mathrm{d} \boldsymbol{k},
$$

where $\tilde{\phi}^{S}(\boldsymbol{k})$ is the spatial Fourier transform of $\phi^{S}(\boldsymbol{x})$. We note that (3.5) is slightly less accurate in the free-decay case since the spectra slowly evolve in the quasi-stationary state. However, the evolution rate of the spectra is much smaller than the energy flux so that the associated error is negligible.

The scaling between $E_{i n}$ and $P^{1 / 3}$ is plotted in figure 4 for the free-decay case and the two forcing cases. The variable $P^{1 / 3}$ is used for the horizontal axis so that the WTT scaling $E_{\text {in }} \sim P^{1 / 3}$ becomes a straight line in the figure. We see that, at high nonlinearity level, the scaling in all cases approaches the WTT scaling, although the range of consistency is longer in the free-decay case and the broad-band forcing case. With the decrease of nonlinearity, the scaling deviates from the WTT prediction with a smaller value of $P$ for given $E_{i n}$. This indicates a reduced capacity of energy cascade with the reduction of nonlinearity. As the nonlinearity level is further decreased, all curves approach states with $P \rightarrow 0$ and finite $E_{i n}$, suggesting the formation of 'frozen turbulence', which is previously (only) introduced for capillary waves (Pushkarev \& Zakharov 2000; Pan \& Yue 2014). This result is remarkable because of the existence of (sparse) exact resonances for gravity waves on a discrete grid of $\boldsymbol{k}$ (rational torus), in contrast to capillary waves. Before this study it is not clear whether the exact quartet resonances of gravity waves can be connected to result in a cascade (Kartashova, Nazarenko \& Rudenko 2008), although this has been demonstrated to be possible in MMT turbulence (Hrabski \& Pan 2020). The results here suggest that the energy cascade should not be expected under the current wavenumber range in spite of the existence of energy transfer within a small number of resonant quartets. A more detailed study can be performed considering the kinematic expansions of exact quartet interactions (e.g. Lvov et al. 2006; Hrabski et al. 2021).

In the following, we study hypothetical physical mechanisms concerning bound waves and the finite-size effect, which may lead to the spectral behaviours presented in $\S \S 3.1$ and 3.2 .

\subsection{Bound waves}

In this section, we study the effect of bound waves, which is argued as a major factor influencing spectral behaviour in previous experiments (Michel et al. 2018). Here we generally define bound waves as wave components that do not satisfy the linear dispersion relation, no matter whether they are generated by carrier-wave distortion (Plant et al. 1999, 2004), superharmonics (Herbert et al. 2010; Cobelli et al. 2011; Michel et al. 2018) or other mechanisms (Longuet-Higgins 1992; Campagne et al. 2019) proposed in previous literature. In fact, as we show in $\S 3.3 .1$, all bound waves in a periodic-domain simulation can be interpreted through non-resonant nonlinear interactions. This definition distinguishes bound waves from free waves which satisfy the linear dispersion relation (or lie in its vicinity). To separate bound waves from the wave field, it is necessary to conduct a spatiotemporal analysis and obtain the wavenumber-frequency spectrum $S_{\eta}(k, \omega)$, 


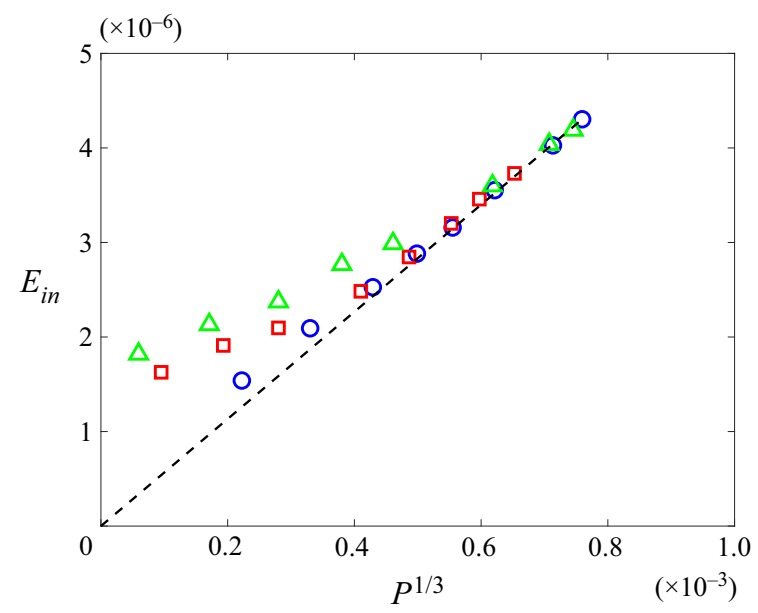

Figure 4. Plots of the energy density in the inertial range $E_{\text {in }}$ as a function of $P^{1 / 3}$ for free-decay (o, blue), broad-band forcing $(\square$, red) and narrow-band forcing $(\triangle$, green) cases, compared with the prediction of WTT $E_{\text {in }} \sim P^{1 / 3}(---)$.

defined as

$$
S_{\eta}(k, \omega)=\int_{0}^{2 \pi}|\tilde{\eta}(\boldsymbol{k}, \omega)|^{2} k \mathrm{~d} \theta
$$

where $\tilde{\eta}(\boldsymbol{k}, \omega)$ is the spatiotemporal Fourier transform of $\eta(\boldsymbol{x}, t)$ :

$$
\tilde{\eta}(\boldsymbol{k}, \omega)=\iiint_{\left[0, T_{L}\right] \times[0,2 \pi] \times[0,2 \pi]} \eta(\boldsymbol{x}, t) h_{T}(t) \exp (-\mathrm{i}(\boldsymbol{k} \cdot \boldsymbol{x}-\omega t)) \mathrm{d} \boldsymbol{x} \mathrm{d} t,
$$

with $h_{T}(t)$ the Tukey window (Bloomfield 2004) of length $T_{L}=20 T_{p}$, the time duration of the collected data within $1480 T_{p}-1500 T_{p}$ at the stationary state.

\subsubsection{Generation mechanisms}

The wavenumber-frequency spectra $S_{\eta}(k, \omega)$ are plotted in figure 5 for narrow-band forcing with low and high nonlinearity levels, and broad-band forcing with low and high nonlinearity levels. The results for free-decay cases are not shown since they are somewhat similar to those for the broad-band forcing case. In all cases, we (seem to) observe significant amount of energy away from the linear dispersion relation (red curve in each panel), indicating the presence of bound waves in the simulations. In addition, the plot for narrow-band forcing at low nonlinearity (figure $5 a$ ) shows discrete peaks of bound-wave components, a visually distinct pattern from the other panels. With respect to the results in $\S 3.1$, these discrete peaks correspond to the peaks in wavenumber spectra with low nonlinearity in figure $2(d)$. As marked by red dots in figure 5(a), these peaks can be quantified as superharmonics of the peak mode $\left(\omega_{p}, k_{p}\right)$ of carrier waves, in the form of

$$
\left(\omega_{b}, k_{b}\right)=\left(n \omega_{p}, n k_{p}\right), \quad n=2,3,4, \ldots
$$

The phase velocity for all bound-wave components, described by (3.8), can be computed as $\omega_{b} / k_{b}=\omega_{p} / k_{p}$, which is the same as the phase velocity of the peak mode of carrier waves. Therefore, the bound waves can be considered as non-dispersive, i.e. they are 'bound' to 
Numerical investigation of surface gravity wave turbulence
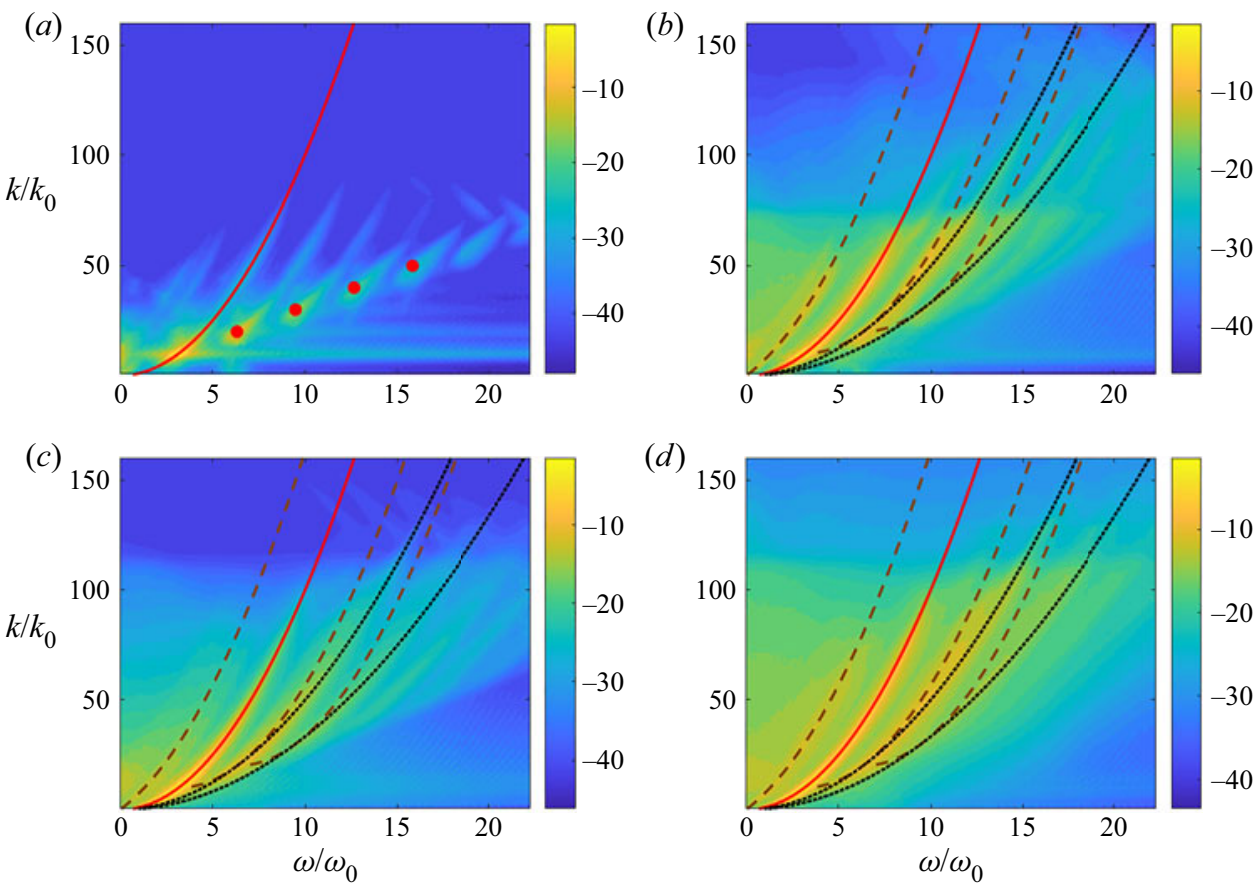

Figure 5. Normalized wavenumber-frequency spectra $S_{\eta}(\omega, k) / S_{\eta}\left(\omega_{p}, k_{p}\right)$ in $\log$ scale for narrow-band forcing cases with $(a) \epsilon=0.059$ and $(b) \epsilon=0.148$ and for broad-band forcing cases with $(c) \epsilon=0.061$ and $(d) \epsilon=0.145$. The linear dispersion relation is marked by $(-$, red). In $(a)$, the peak modes are marked by $(\bullet$, red) computed from (3.8) with $n=2,3,4,5$. In $(b-d)$, the lines corresponding to (3.9) with $m=2,3$ are indicated by $\cdot \cdot$, and the lines corresponding to (3.10) with $l=-1,1,2$ are indicated by (-- , brown).

the carrier wave as the latter travels. This behaviour agrees with some of the classical views of bound waves described in Lake \& Yuen (1978) and Plant (2003).

For cases in figure $5(b-d)$, however, the bound-wave patterns are dramatically different from that in 5(a). It is clear from the plots that these bound-wave components are not necessarily non-dispersive, which is consistent with other general views of bound waves (e.g. Phillips 1981). In these cases, we observe several bound-wave branches (in contrast to the main branch of linear dispersion relation) on the spectra $S_{\eta}(\omega, k)$. These bound-wave branches can be explained through two different mechanisms discussed below.

The first mechanism corresponds to the superharmonics of all free waves, i.e. modes $(\omega, k)$ in the main (carrier) branch of the linear dispersion relation, satisfying

$$
\left(\omega_{b}, k_{b}\right)=(m \omega, m k), \quad m=2,3,4, \ldots
$$

The second mechanism corresponds to bound waves generated by the (non-resonant) interactions between the harmonics of peak mode $\left(\omega_{p}, k_{p}\right)$ and an arbitrary free wave $(\omega, k)$ in the main branch, satisfying

$$
\left(\omega_{b}, k_{b}\right)=\left(\omega+l \omega_{p}, k+l k_{p}\right), \quad l= \pm 1, \pm 2, \pm 3, \ldots
$$

The curves described by (3.9) and (3.10) are marked in figure 5(b-d) respectively by black solid lines and brown dashed lines. The two mechanisms co-explain the superbranches of bound waves on the right of the main branch, as they both contribute to the energy in each of the visible superbranches. The second mechanism with 


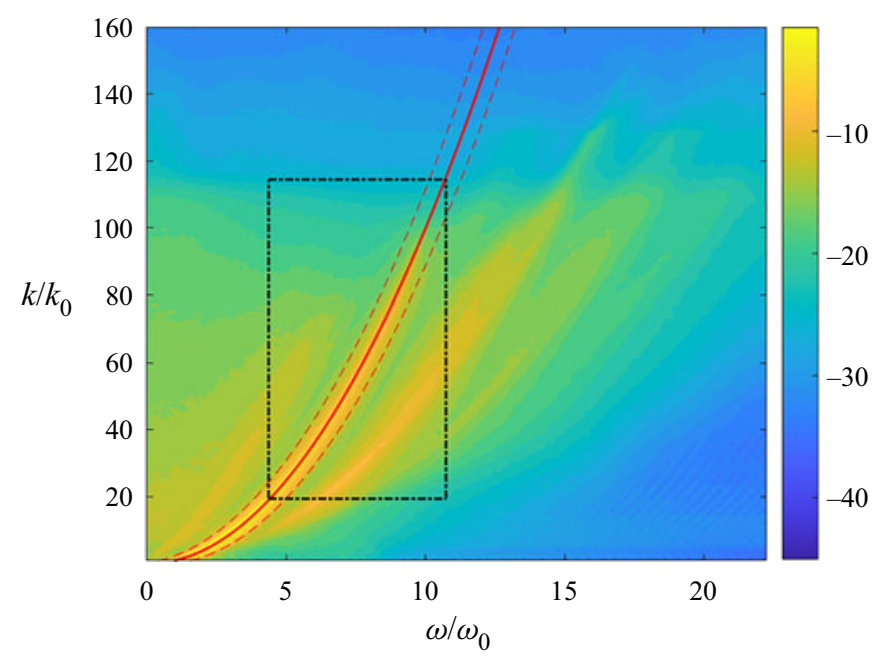

Figure 6. A typical illustration for the separation of free and bound waves in a wavenumber-frequency spectrum. The region of $\left[\omega_{k}-\delta \omega, \omega_{k}+\delta \omega\right]$ defined in (3.11) is marked by $(---$, red). The region bounded by - - - corresponds to the inertial ranges $\left[\omega_{c}, \omega_{d}\right] \times\left[k_{c}, k_{d}\right]$ used in (3.12).

$l=-1$ further explains the sub-branches of bound waves on the left of the main branch. We remark that the two mechanisms (3.9) and (3.10) are also separately observed in experiments of Herbert et al. (2010), Cobelli et al. (2011) and Campagne et al. (2019). Our results here provide a more comprehensive view of bound waves: we need to consider the property of carrier waves to distinguish cases in figures $5(a)$ and $5(b-d)$, and consider combined mechanisms (3.9) and (3.10) for the explanation in the latter case.

\subsubsection{Effects on wave spectra}

Our next goal is to separate bound waves and free waves to elucidate their relative importance to the wave turbulence of gravity waves. The algorithm for the separation is illustrated in figure 6, which shows the definition of a free-wave finite band (by dashed line) in the vicinity of the linear dispersion relation. Specifically, this finite band is generated through a filter (similar to Campagne et al. 2019):

$$
f(\boldsymbol{k}, \omega) \equiv \begin{cases}1, & \left|\omega-\omega_{k}\right| \leq \delta \omega \\ 0, & \left|\omega-\omega_{k}\right|>\delta \omega\end{cases}
$$

where $\delta \omega=0.6 \omega_{0}$ is a parameter characterizing the width of the free-wave band, with $\omega_{0}=1$ the fundamental frequency in the domain. We have tested that this choice of $\delta \omega$ lies in a stationary range in terms of the results discussed below, i.e. reducing or increasing it results in respectively insufficient free-wave energy or contamination by bound-wave branches. For the separation, we apply the filter (3.11) directly to $\tilde{\eta}(\boldsymbol{k}, \omega)$ and obtain the free-wave and bound-wave components as $\tilde{\eta}_{f}(\boldsymbol{k}, \omega)=f(\boldsymbol{k}, \omega) \tilde{\eta}(\boldsymbol{k}, \omega)$ and $\tilde{\eta}_{b}(\boldsymbol{k}, \omega)=\tilde{\eta}(\boldsymbol{k}, \omega)-\tilde{\eta}_{f}(\boldsymbol{k}, \omega)$. Then we compute the free-wave spectra $S_{\eta}^{f}(k, \omega)$, $S_{\eta}^{f}(k)$ and bound-wave spectra $S_{\eta}^{b}(k, \omega), S_{\eta}^{b}(k)$ likewise using (3.6) and (3.1). We note that it is also possible to directly apply the filter $(3.11)$ to $S_{\eta}(k, \omega)$ for the separation, but our operation (with respect to $\tilde{\eta}$ ) is desirable due to the study that is discussed in $\S 3.4$. 

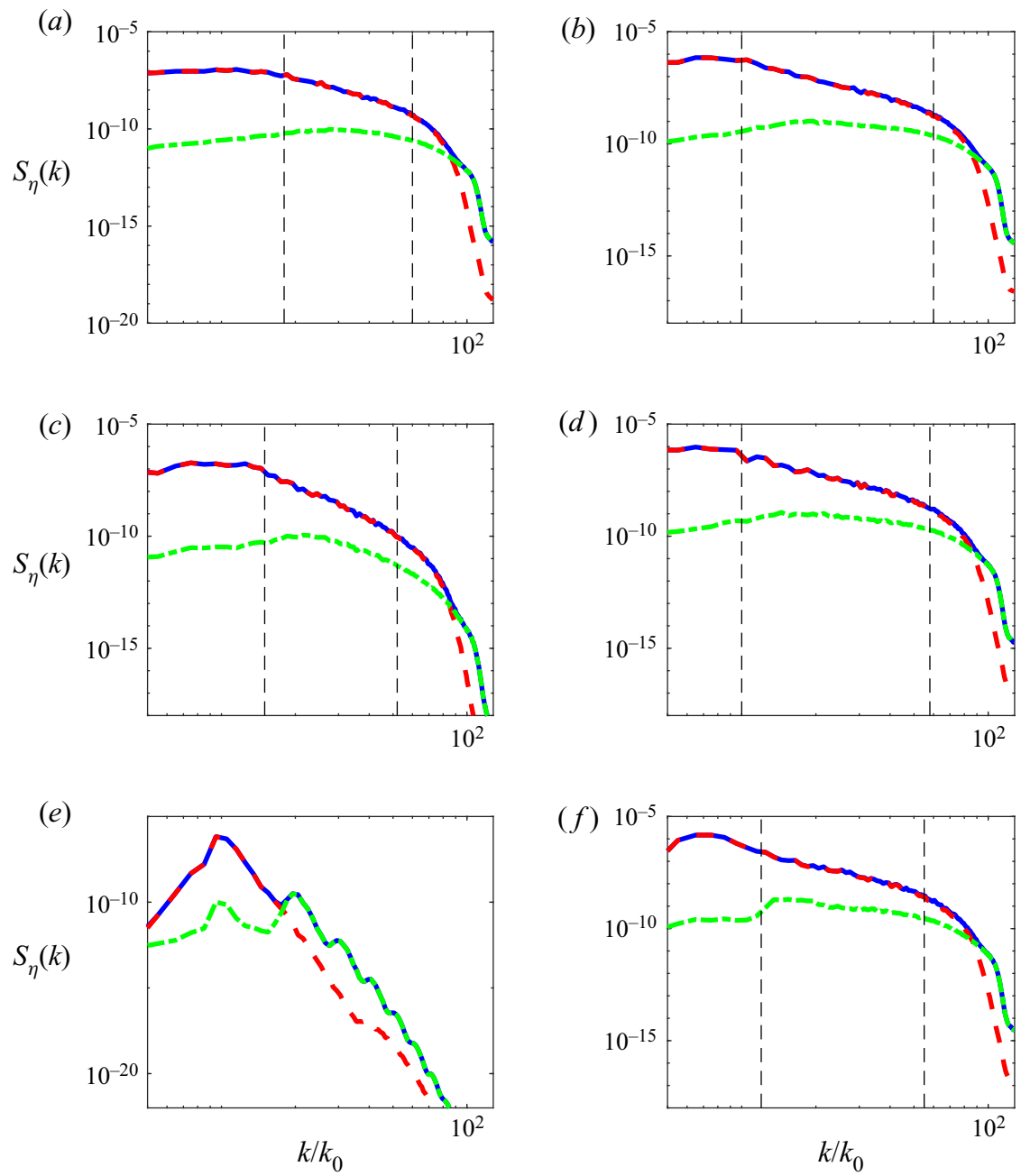

Figure 7. Plots of $S_{\eta}^{f}(k)\left(---\right.$, red), $S_{\eta}^{b}(k)\left(-\cdots\right.$, green) and $S_{\eta}(k)(-$, blue) in free-decay cases with $(a)$ $\epsilon=0.068$ and $(b) \epsilon=0.151$; broad-band forcing cases with $(c) \epsilon=0.071$ and $(d) \epsilon=0.145$; narrow-band forcing cases with $(e) \epsilon=0.059$ and $(f) \epsilon=0.148$. The boundaries of the power-law ranges are indicated by --- , except for $(e)$ where discrete peaks are observed.

The free-wave and bound-wave wavenumber spectra are shown in figure 7 for all three (free-decay and broad-band and narrow-band forcing) cases at high and low nonlinearity levels. In most cases, we find that the power-law ranges of the spectra are dominated by free-wave components with their energy at least one order of magnitude higher than those of the bound-wave components. The only exception is the case with narrow-band forcing at low nonlinearity level shown in figure 7(e), where bound waves dominate most of the range above $k \approx 17$ exhibiting discrete peaks. On the other hand, waves at sufficiently small scales in the dissipation range are dominated by bound waves in all cases, but their influences to the inertial-range spectra are not significant.

The general behaviour of bound waves in figure 7 suggests that they are not the major factor causing the steepening of the spectra except in the narrow-band forcing case. This point will be made more clear after further quantifying the fraction of bound waves in the 


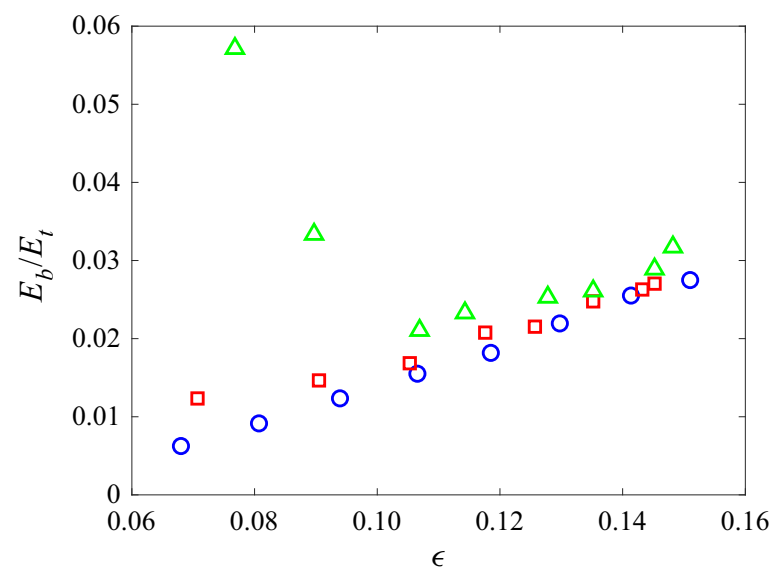

Figure 8. The proportions of bound-wave energy $E_{b} / E_{t}$ as functions of $\epsilon$ for the free-decay (o, blue), broad-band forcing $(\square$, red) and narrow-band forcing $(\triangle$, green) cases.

wave field. For this purpose, we define the bound-wave energy in the inertial range as

$$
E_{b} \equiv \int_{\omega_{c}}^{\omega_{d}} \int_{k_{c}}^{k_{d}} S_{\eta}^{b}(k, \omega) \mathrm{d} k \mathrm{~d} \omega,
$$

where $\left[k_{c}, k_{d}\right]$ and $\left[\omega_{c}=k_{c}^{1 / 2}, \omega_{d}=k_{d}^{1 / 2}\right]$ are a box describing the limits of inertial range as shown in figure 6. We quantify the fraction of bound waves using the ratio of $E_{b} / E_{t}$, where $E_{t}$ is the total wave energy in the inertial range computed in a similar way to (3.12) but with $S_{\eta}(k, \omega)$ replacing $S_{\eta}^{b}(k, \omega)$.

Figure 8 summarizes the quantity $E_{b} / E_{t}$ at different nonlinearity levels for all three cases. For the broad-band forcing and free-decay cases, we see that the fraction of bound-wave energy consistently decreases with a decrease of nonlinearity level. This provides a clear evidence that the steepening of the spectra with the decrease of nonlinearity is not caused by bound waves in these cases. For the narrow-band forcing case, the fraction of bound-wave energy is consistently larger than those in the other two cases (at all nonlinearity levels). In addition, with a decrease of nonlinearity, a transition occurs at a critical nonlinearity level $\left(\epsilon_{c} \approx 0.11\right)$, below which $E_{b} / E_{t}$ increases with a decrease of $\epsilon$. In general, we expect the critical level $\epsilon_{c}$ to increase with a decrease of the forcing bandwidth. (For the broad-band forcing case considered here, the frozen turbulence regime is reached first with a decrease of nonlinearity, so $\epsilon_{c}$ does not exist in this case.) The critical level $\epsilon_{c}$ is consistent with the transition in figure 3 , below which a much more rapid steepening of the spectral slope is observed for the narrow-band forcing case. We thus conclude that the presence of bound waves explains the largest steepening rate of the spectra at low nonlinearity levels in the narrow-band forcing case. Moreover, the significant fraction of bound waves in the narrow-band forcing case also provides an explanation of the rapid deviation from $E_{i n} \sim P^{1 / 3}$ shown in figure 4 , since bound waves account for energy which does not follow the WTT cascade pathway.

We finally remark that while the transition at $\epsilon_{c}$ in figure 8 is consistent with the transition to rapid variation of $\alpha$ in figure 3, the percentage of bound waves remains not significant for the range of nonlinearity levels considered in the two figures. As a result, computing the spectral slope from $S_{\eta}^{f}(k)$ provides almost the same values as those shown in 3 , as evidenced from figure 7 for many cases (which is also true for the narrow-band forcing 
cases at nonlinearity levels associated with power-law spectra). Physically, this suggests that bound waves cannot be simply considered as linear superpositions on free waves. Instead, their nonlinear interactions with free waves can be important in understanding the evolution and dynamics of the wave fields.

\subsection{Finite-size effect}

In order to further understand the steepening of spectra and reduced energy flux capacity at low nonlinearity levels, we investigate another hypothetical mechanism of the finite-size effect. In general, the finite-size effect arises due to the violation of the assumption of the infinite domain in WTT (Pushkarev \& Zakharov 2000; Lvov et al. 2006; Nazarenko 2006). In the framework of the WTT kinetic equation, the energy cascade is enabled by modes on the continuous resonant manifold satisfying (for gravity waves)

$$
\begin{aligned}
& \boldsymbol{k}_{1}+\boldsymbol{k}_{2}=\boldsymbol{k}_{3}+\boldsymbol{k}, \\
& \omega_{1}+\omega_{2}=\omega_{3}+\omega,
\end{aligned}
$$

where $\omega_{i}^{2}=k_{i}(i=1,2,3)$. In a finite domain, discreteness of wavenumber and frequency (imposed by the domain boundary) reduces the manifold defined by (3.13) and (3.14) by limiting the resonances to discrete points. This discreteness effect can be compensated by the nonlinear broadening which allows the occurrence of quasi-resonances, characterized by a modification of (3.14) as

$$
\left|\omega_{1}+\omega_{2}-\omega_{3}-\omega\right|<\Delta \omega,
$$

with $\Delta \omega$ a nonlinear broadening parameter depending on the nonlinearity level.

One way to quantify the finite-size effect (in particular to measure the nonlinear broadening and interaction strength) of gravity waves is through a tri-coherence analysis (see e.g. Pan \& Yue (2017) for a bi-coherence study of capillary waves). We first define a tri-coherence function

$$
T\left(\boldsymbol{k}, \boldsymbol{k}_{1}, \boldsymbol{k}_{2}\right) \equiv \frac{\left|\left\langle\tilde{\eta}_{f}\left(\boldsymbol{k}_{1}, t\right) \tilde{\eta}_{f}\left(\boldsymbol{k}_{2}, t\right) \tilde{\eta}_{f}^{*}\left(\boldsymbol{k}_{3}, t\right) \tilde{\eta}_{f}^{*}(\boldsymbol{k}, t)\right\rangle\right|}{\left\langle\left|\tilde{\eta}_{f}\left(\boldsymbol{k}_{1}, t\right)\left\|\tilde{\eta}_{f}\left(\boldsymbol{k}_{2}, t\right)\right\| \tilde{\eta}_{f}^{*}\left(\boldsymbol{k}_{3}, t\right)\right|\left|\tilde{\eta}_{f}^{*}(\boldsymbol{k}, t)\right|\right\rangle},
$$

where $\boldsymbol{k}_{3} \equiv-\boldsymbol{k}+\boldsymbol{k}_{1}+\boldsymbol{k}_{2},\langle\cdot\rangle$ denotes the time average (for stationary states) and an asterisk denotes the complex conjugate. Here $\tilde{\eta}_{f}(\boldsymbol{k}, t)$ corresponds to the free-wave modes, and is computed by the spatial Fourier transform of $\eta_{f}(\boldsymbol{x}, t)$ with the latter obtained after applying the filter (3.11) to the wavenumber-frequency spectra. By definition, the function $T\left(\boldsymbol{k}, \boldsymbol{k}_{1}, \boldsymbol{k}_{2}\right)$ continuously varies between 0 and 1 . In particular, $T$ takes larger values for quartets closer to exact resonance, with the maximum value of 1 achieved for exact resonances among $\boldsymbol{k}, \boldsymbol{k}_{1}, \boldsymbol{k}_{2}$ and $\boldsymbol{k}_{3}$ satisfying (3.14). In (3.16), we use $\tilde{\eta}_{f}$ instead of $\tilde{\eta}$ for the evaluation to remove the contamination by bound waves since they result in noisy values of $T$ not lying in the vicinity of the resonant manifold (as found in previous work (Pan \& Yue 2017)). This operation is also compatible with findings in $\S 3.3$ that bound waves do not contribute to the general steepening of the spectra (except in the narrow-band forcing case).

To facilitate the visualization of $T$, we fix $\boldsymbol{k}_{1}=(40,40)$ and $\boldsymbol{k}_{2}=(20,-40)$ so that $T(\boldsymbol{k})$ with $\boldsymbol{k}=\left(k_{x}, k_{y}\right)$ can be shown by two-dimensional contour plots. Figure 9 shows such plots of $T(\boldsymbol{k})$ in the broad-band forcing case at three different nonlinearity levels. The results for the narrow-band forcing and free-decay cases are not included due to their similarity to the presented results (the quantification of broadening and interaction 

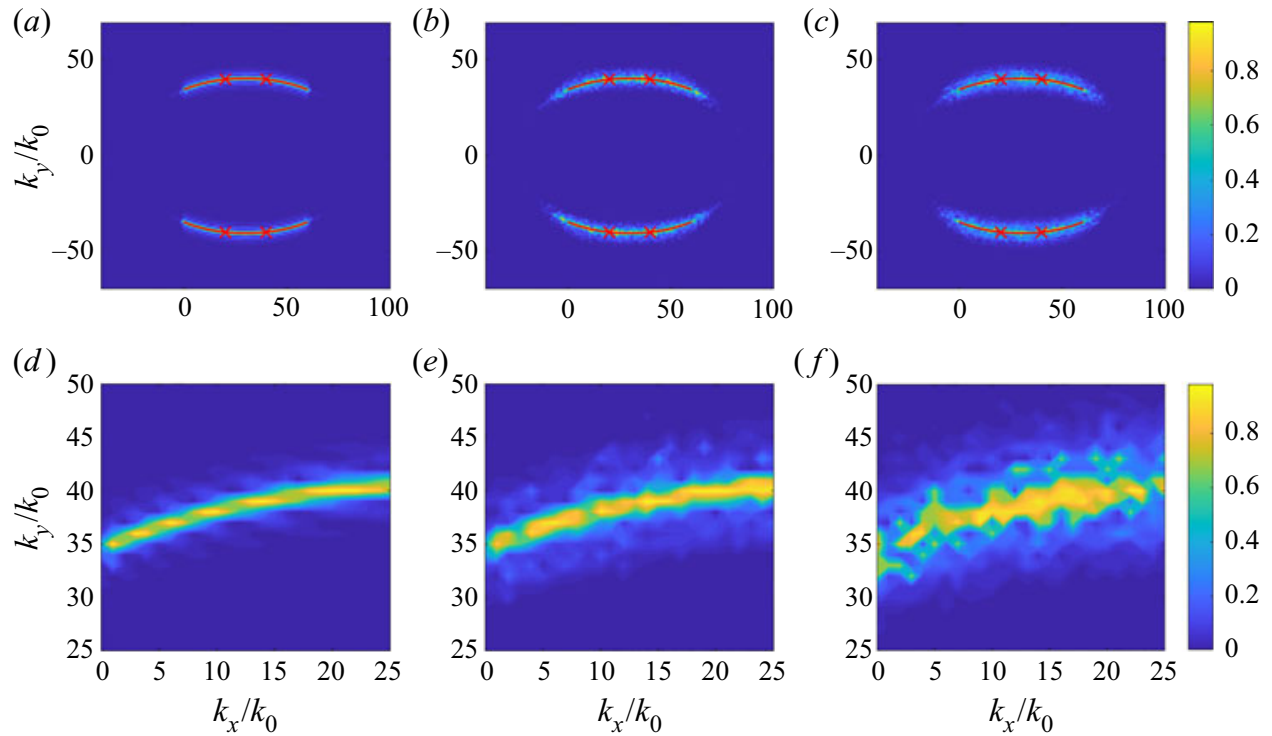

Figure 9. The tri-coherence $T\left(\boldsymbol{k}, \boldsymbol{k}_{1}, \boldsymbol{k}_{2}\right)$ with $\boldsymbol{k}_{1}=(40,40), \boldsymbol{k}_{2}=(20,-40)$ for broad-band forcing cases with $(a) \epsilon=0.071,(b) \epsilon=0.118$ and $(c) \epsilon=0.145$. The continuous resonant manifold is plotted by $(-$, red $)$. The discrete resonant solutions with $k \in(\mathbb{Z}, \mathbb{Z})$ are marked by $(\times$, red). The data for generating these figures are collected from $t=1460 T_{p}$ to $t=1500 T_{p}$ in the stationary state. $(d-f)$ Zoom-in views of $(a-c)$ respectively.

strength for all cases is presented later). Also shown in figure 9 is the continuous resonant manifold (red lines) satisfying (3.13) and (3.14), and the discrete resonant points (red crosses) with $\boldsymbol{k}$ only taking integer values. In particular, to compute the resonant manifold we need to consider the wave travelling direction and the complex conjugate relation for real functions, with details presented in Appendix A.

From figure $9(a-c)$ we can see that all significant values of $T$ are concentrated close to the resonant manifold (as a result of using $\tilde{\eta}_{f}$ in (3.16)). It is also clear that the nonlinear broadening is visibly wider at a higher nonlinearity level compared with that at a lower nonlinearity level. The finite-size effect can be further visualized through the zoom-in views in figure $9(d-f)$. At low nonlinearity, figure $9(d)$ shows discrete peaks of $T \sim O(1)$ at only a number of grid points $\left(k_{x}, k_{y}\right)$ lying on or very close to the resonant manifold. This is a direct result of the finite-size effect, as it is in contrast to the situation of $T$ taking uniform values of 1 on the resonant manifold that can be expected in an infinite domain. With an increase of nonlinearity level from figure $9(d)$ to $9(f)$, the broadening of the interaction width overcomes the discreteness in $T$, alleviating its destructing effect on the nonlinear interactions.

In addition, the discrete resonant points seem to be sparse on the continuous manifold, with all four points in each panel of figure 9 as trivial quartet solutions (either with repetition or symmetry with given vectors of $\boldsymbol{k}_{1}$ and $\boldsymbol{k}_{2}$ ). This sparsity can be further demonstrated by a numerical analysis which identifies no non-trivial solution with stretching of the current resonant manifold (see Appendix B). This problem has also been considered analytically and numerically in Kartashova (2006) and Lvov et al. (2006), which reach a consistent conclusion on the rareness of the discrete solutions except for two special types of collinear quartets and tridents (which, however, do not exist in our case with waves propagating only to the positive $x$ direction). Moreover, the sparsity of discrete solutions in this case is in contrast to the situation of the $\omega=k^{2}$ MMT dispersion relation 

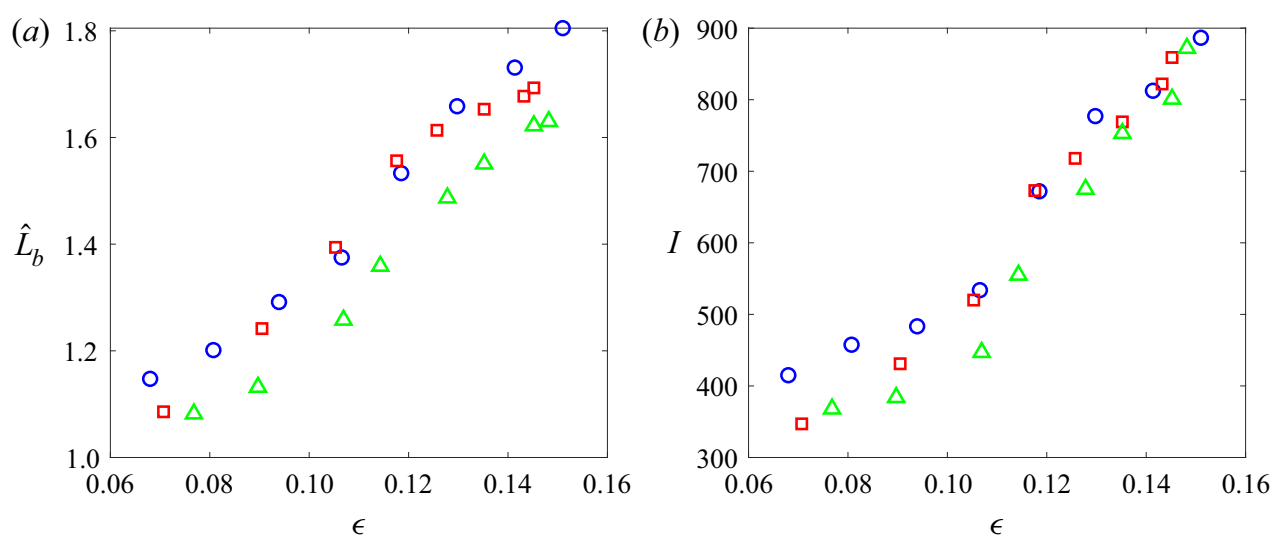

Figure 10. (a) The characteristic length of nonlinear broadening $\hat{L}_{b}$ and $(b)$ the intensity of nonlinear interactions $I$ as functions of $\epsilon$ for the free-decay (०, blue), broad-band forcing ( $\square$, red) and narrow-band forcing $(\triangle$, green) cases.

studied in Hrabski \& Pan (2020). The difference in the structure of the discrete resonant solutions is the key for the gravity-wave spectra and MMT spectra to show completely different behaviours at low nonlinearity, with the former deviating from the WTT spectral slope but the latter approaching the WTT spectral slope.

To further quantify the effect of nonlinearity level on quartet resonances, we define two measures $\hat{L}_{b}$ and $I$ respectively for the broadening width and overall interaction strength. For $\hat{L}_{b}$, we define it as a characteristic width (Pan \& Yue 2017):

$$
\hat{L}_{b} \equiv \frac{\sum_{k}|\hat{\Omega}| T(k)}{\sum_{k} T(k)}
$$

where $|\hat{\Omega}|$ is the normalized frequency mismatch given by $|\hat{\Omega}|=|\Omega| / k^{-1 / 2}$ with $\Omega \equiv$ $\omega_{1}+\omega_{2}-\omega_{3}-\omega_{k}$ and the denominator $k^{-1 / 2}$ estimating the frequency discreteness at $k$ associated with the wavenumber spacing. In (3.17), the summation is for all the grid points of $\boldsymbol{k}$. Thus, $\hat{L}_{b}$ measures the nonlinear broadening around the exact solutions by the first moment of $T(\boldsymbol{k})$.

We further define $I$ as

$$
I \equiv \sum_{k} T(\boldsymbol{k})
$$

which measures the overall interaction strength by summing up contributions from both resonant and quasi-resonant interactions. We remark that $I$ provides supplemental information to $L_{b}$, because $L_{b}$ itself does not quantify the dynamics of energy transfer, i.e. a larger $L_{b}$ does not mean a stronger energy transfer if the function $T$ becomes more discrete in the interaction broadening region. In contrast, $I$ provides a measure of the overall interaction strength accounting for both the finite-size effect and the nonlinear broadening. Another improved quantification of the interaction strength of exact and quasi-resonances through a quartet-level decomposition of the energy flux can be found in Hrabski \& Pan (2021).

The characteristic width $\hat{L}_{b}$ and the interaction strength $I$ are plotted as functions of $\epsilon$ for all cases in figure 10. We can observe a general increase of $\hat{L}_{b}$ and $I$ for increasing $\epsilon$, indicating a wider nonlinear broadening and a larger interaction strength with more 


\section{Z. Zhang and Y. Pan}

quasi-resonances alleviating the finite-size effect. We remark that this clear behaviour of $\hat{L}_{b}$ and $I$ is only possible to resolve by using $\tilde{\eta}_{f}$ to evaluate the tri-coherence. The general trend of $\hat{L}_{b}$ with respect to $\epsilon$ seems to be consistent with the nonlinear detuning (or correction) of the dispersion relation introduced in Stuhlmeier \& Stiassnie (2019), but the exact relation between the two quantities is yet to be understood. The reduction of the nonlinear broadening and the interaction strength with decreasing nonlinearity is consistent with the steepening of spectra and the reduction of energy flux capacity discussed in $\S \S 3.1$ and 3.2. Therefore, we conclude that the finite-size effect is a major contributor to the spectral behaviours at low nonlinearity levels (especially for cases with sufficient spectral bandwidth). Finally, the values of $\hat{L}_{b}$ and $I$ in the narrow-band forcing case are slightly smaller than in the other two cases for all nonlinearity levels, probably because of the higher fraction of bound waves (which leads to a larger $\epsilon$ than the other two cases, but not larger $\hat{L}_{b}$ or $I$ ).

\section{Conclusions}

We conduct numerical simulation of Euler equations to study the surface gravity wave turbulence in three representative conditions, namely free-decay, narrow-band forcing and broad-band forcing turbulence. In all cases, We find that the scalings of the wave spectra with wavenumber and energy flux both approach the WTT solution at sufficiently high nonlinearity levels. With a decrease of nonlinearity level, steeper spectra and reduced energy flux capacity can be observed indicating the deviation from WTT, with the largest deviation rate found in the narrow-band forcing case. Two hypothetical mechanisms for bound waves and finite-size effect to explain these spectral variations are investigated. For bound waves, we elucidate their generation mechanisms through a spatiotemporal analysis (which generalizes the previous study of this topic) and find that their fraction generally decreases with a decrease of nonlinearity level except for the narrow-band forcing case. This suggests that bound waves only account for the rapid deviation from WTT in the narrow-band forcing case (but not for the other two cases). For the finite-size effect, we perform a tri-coherence analysis and find that both the nonlinear broadening and interaction strength decrease with a decrease of nonlinearity level, which accounts for the deviation from WTT at low nonlinearity levels in all cases of our simulation. We finally remark that cautions have to be taken in applying these numerical findings to experiments due to the additional complexity inevitably involved in the latter.

Declaration of interests. The authors report no conflict of interest.

Author ORCIDs.

(D) Yulin Pan https://orcid.org/0000-0002-7504-8645.

\section{Appendix A. Computation of resonant manifold in figure 9}

We start by considering a (quasi-)stationary wave field described by

$$
\eta(x, t)=\sum_{i} A_{i} \exp \left(\mathrm{i}\left(\kappa_{i} \cdot x-\omega_{i} t\right)\right)+A_{i}^{*} \exp \left(-\mathrm{i}\left(\kappa_{i} \cdot x-\omega_{i} t\right)\right),
$$

where $A_{i}=\left|A_{i}\right| \mathrm{e}^{\mathrm{i} \phi_{i}} \in \mathbb{C}$ and $\omega_{i} \in \mathbb{R}^{+}$. Since the wave fields in our simulations generally only contain waves travelling to the positive $x$ direction (due to the setting of initial condition and forcing), for each mode in (A1) we have $\kappa_{i x} \cdot \omega_{i}>0$ so that $\kappa_{i}=\left(\kappa_{i x}, \kappa_{i y}\right) \in$ $\left(\mathbb{R}^{+}, \mathbb{R}\right)$. 

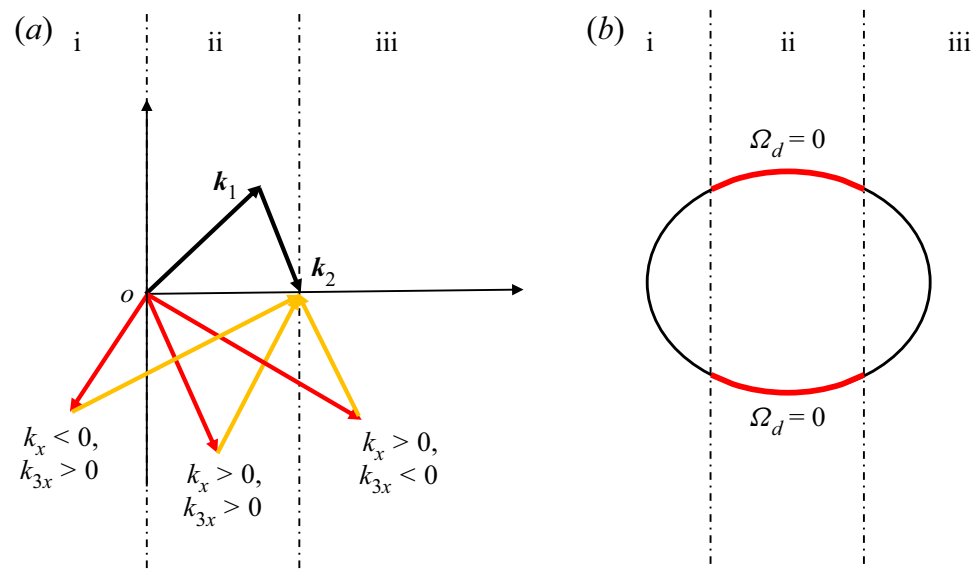

Figure 11. (a) Sketches of the three situations under discussion. Vectors $\boldsymbol{k}_{1}$ and $\boldsymbol{k}_{2}$ are indicated in the figure; $\boldsymbol{k}$ and $\boldsymbol{k}_{3}$ are denoted by red and yellow arrows. The two vertical lines mark the boundaries of situations (i), (ii) and (iii) in the discussion. (b) The solution in situation (ii) as denoted by red lines (ellipse bounded by the two vertical lines).

Considering $\tilde{\eta}(\boldsymbol{k}, t)$ with $\boldsymbol{k} \in(\mathbb{R}, \mathbb{R})$ as the spatial Fourier transform of $\eta(\boldsymbol{x}, t)$, we can see that

$$
\tilde{\eta}\left(\boldsymbol{k}_{i}, t\right)= \begin{cases}A_{i} \mathrm{e}^{-\mathrm{i} \omega_{i} t}, & k_{i x}>0, \\ A_{i}^{*} \mathrm{e}^{\mathrm{i} \omega_{i} t}, & k_{i x}<0 .\end{cases}
$$

Substituting (A2) into (3.16), we obtain

$$
T=\left|\left\langle\exp \left(\mathrm{i}\left(-\Omega_{d} t+\Phi_{d}\right)\right)\right\rangle\right|,
$$

where $\Phi_{d}=\phi_{1}+\phi_{2}-\phi_{3}-\phi$ and

$$
\Omega_{d}=\operatorname{sgn}\left(k_{1 x}\right) \omega_{1}+\operatorname{sgn}\left(k_{2 x}\right) \omega_{2}-\operatorname{sgn}\left(k_{3 x}\right) \omega_{3}-\operatorname{sgn}\left(k_{x}\right) \omega,
$$

with

$$
\operatorname{sgn}(x)= \begin{cases}1, & x>0, \\ -1, & x<0 .\end{cases}
$$

In our case, we have $k_{1 x}=40>0, k_{2 x}=20>0$ and $k_{3 x}=k_{1 x}+k_{2 x}-k_{x}=60-$ $k_{x}$, so that (A4) is reduced to $\Omega_{d}=\omega_{1}+\omega_{2}-\operatorname{sgn}\left(60-k_{x}\right) \omega_{3}-\operatorname{sgn}\left(k_{x}\right) \omega$. We are interested in the case of $\Omega_{d}=0$, which results in $T=1$ indicating the resonant manifold. Whether $\Omega_{d}=0$ can be realized needs to be discussed in the following three situations (summarized in figure 11a):

(i) For $k_{x}<0$, we need $\Omega_{d}=\omega_{1}+\omega_{2}-\omega_{3}+\omega=0$ which has no solution (for $\omega \in$ $\left.\mathbb{R}^{+}\right)$on the $\left(k_{x}, k_{y}\right)$ plane.

(ii) For $0<k_{x}<60$, we need $\Omega_{d}=\omega_{1}+\omega_{2}-\omega_{3}-\omega=0$ which has solutions shown as an ellipse bounded by $k_{x}=0$ and $k_{x}=60$ in figure $11(b)$. These solutions correspond to the resonant manifold shown in figure 9 .

(iii) For $k_{x}>60$, we have $\Omega_{d}=\omega_{1}+\omega_{2}+\omega_{3}-\omega=0$ which has no solution on the $\left(k_{x}, k_{y}\right)$ plane.

In summary, the resonant manifold shown in figure 9 corresponds to the second case above, which is the only possibility to have solutions in $\Omega_{d}=0$ for a wave field travelling to the positive $x$ direction. 


\section{Z. Zhang and Y. Pan}

\section{Appendix B. Sparsity of the discrete resonant solutions}

We count the number of discrete resonant solutions as in figure 9, but with stretched $\boldsymbol{k}_{1}$ and $\boldsymbol{k}_{2}$. The purpose is to demonstrate the sparsity of discrete resonances with the expansion of the resonant manifold. In particular, we define $\boldsymbol{k}_{1}=\lambda(40,40)$ and $\boldsymbol{k}_{2}=\lambda(20,-40)$, where $\lambda$ is a factor of stretching, and compute the number of exact resonances $N$ as a function of $\lambda$. For $\lambda$ varying from 1 to 10 , our numerical searching algorithm gives $N=4$ always, indicating that no non-trivial solution can be identified. While this numerical study is presented for $\boldsymbol{k}_{1}$ and $\boldsymbol{k}_{2}$ in particular directions, we have also tested other directions to confirm that the exact resonances are indeed sparse for gravity waves.

\section{REFERENCES}

Aubourg, Q. \& Mordant, N. 2016 Investigation of resonances in gravity-capillary wave turbulence. Phys. Rev. Fluids 1 (2), 023701.

Bloomfield, P. 2004 Fourier Analysis of Time Series: An Introduction. John Wiley \& Sons.

Campagne, A., Hassaini, R., Redor, I., Valran, T., Viboud, S., Sommeria, J. \& Mordant, N. 2019 Identifying four-wave-resonant interactions in a surface gravity wave turbulence experiment. Phys. Rev. Fluids 4 (7), 074801.

Cobelli, P., Przadka, A., Petitjeans, P., Lagubeau, G., Pagneux, V. \& Maurel, A. 2011 Different regimes for water wave turbulence. Phys. Rev. Lett. 107 (21), 214503.

Deike, L., Berhanu, M. \& FAlCON, E. 2014 Energy flux measurement from the dissipated energy in capillary wave turbulence. Phys. Rev. E 89 (2), 023003.

Deike, L., Miquel, B., Gutiérrez, P., Jamin, T., Semin, B., Berhanu, M., Falcon, E. \& Bonnefoy, F. 2015 Role of the basin boundary conditions in gravity wave turbulence. J. Fluid Mech. 781, 196-225.

Denissenko, P., Lukaschuk, S. \& NAZARenko, S. 2007 Gravity wave turbulence in a laboratory flume. Phys. Rev. Lett. 99 (1), 014501.

Dommermuth, D.G. \& YuE, D.K.P. 1987 A high-order spectral method for the study of nonlinear gravity waves. J. Fluid Mech. 184, 267-288.

Dyachenko, A.I., Korotkevich, A.O. \& Zakharov, V.E. 2004 Weak turbulent kolmogorov spectrum for surface gravity waves. Phys. Rev. Lett. 92 (13), 134501.

Falcon, E., Laroche, C. \& Fauve, S. 2007 Observation of gravity-capillary wave turbulence. Phys. Rev. Lett. 98 (9), 094503.

FAou, E., Germain, P. \& HANi, Z. 2016 The weakly nonlinear large-box limit of the 2D cubic nonlinear Schrödinger equation. J. Am. Math. Soc. 29 (4), 915-982.

HAMMACK, J.L. \& HENDERSON, D.M. 1993 Resonant interactions among surface water waves. Annu. Rev. Fluid Mech. 25 (1), 55-97.

Herbert, E., Mordant, N. \& FAlCon, E. 2010 Observation of the nonlinear dispersion relation and spatial statistics of wave turbulence on the surface of a fluid. Phys. Rev. Lett. 105 (14), 144502.

HRABSKI, A. \& PAN, Y. 2020 Effect of discrete resonant manifold structure on discrete wave turbulence. Phys. Rev. E 102 (4), 041101.

HrabSKi, A. \& PAN, Y. 2021 On the properties of energy flux in wave turbulence. arXiv:2110.07666.

Hrabski, A., Pan, Y., Staffilani, G. \& Wilson, B. 2021 Energy transfer for solutions to the nonlinear schrödinger equation on irrational tori. arXiv:2107.01459.

ISSENMANN, B. \& FALCON, E. 2013 Gravity wave turbulence revealed by horizontal vibrations of the container. Phys. Rev. E 87 (1), 011001.

KARTASHOVA, E. 2006 Fast computation algorithm for discrete resonances among gravity waves. J. Low Temp. Phys. 145 (1), 287-295.

Kartashova, E., NAZAREnKo, S. \& Rudenko, O. 2008 Resonant interactions of nonlinear water waves in a finite basin. Phys. Rev. E 78 (1), 016304.

Kuznetsov, E.A. 2004 Turbulence spectra generated by singularities. J. Expl Theor. Phys. Lett. 80 (2), 83-89.

LAKE, B.M. \& Yuen, H.C. 1978 A new model for nonlinear wind waves. Part 1. Physical model and experimental evidence. J. Fluid Mech. 88 (1), 33-62.

Longuet-Higgins, M.S. 1992 Capillary rollers and bores. J. Fluid Mech. 240, 659-679.

Lvov, Y.V., NAZAREnko, S. \& Pokorni, B. 2006 Discreteness and its effect on water-wave turbulence. Physica D 218 (1), 24-35. 


\section{Numerical investigation of surface gravity wave turbulence}

Mei, C.C., Stiassnie, M. \& Yue, D.K.-P. 2005 Theory and Applications of Ocean Surface Waves: Nonlinear Aspects, vol. 23. World Scientific.

Michel, G., Semin, B., Cazaubiel, A., Haudin, F., Humbert, T., Lepot, S., Bonnefoy, F., BERHANU, M. \& FALCON, É. 2018 Self-similar gravity wave spectra resulting from the modulation of bound waves. Phys. Rev. Fluids 3 (5), 054801 .

NAZAREnKo, S. 2006 Sandpile behaviour in discrete water-wave turbulence. J. Stat. Mech. 2006 (2), L02002.

Nazarenko, S., Lukaschuk, S., Mclelland, S. \& Denissenko, P. 2010 Statistics of surface gravity wave turbulence in the space and time domains. J. Fluid Mech. 642, 395-420.

Onorato, M., Osborne, A.R., Serio, M., Resio, D., Pushkarev, A., Zakharov, V.E. \& Brandini, C. 2002 Freely decaying weak turbulence for sea surface gravity waves. Phys. Rev. Lett. 89 (14), 144501.

PAN, Y. 2020 High-order spectral method for the simulation of capillary waves with complete order consistency. J. Comput. Phys. 408, 109299.

PAN, Y. \& YUE, D.K.P. 2014 Direct numerical investigation of turbulence of capillary waves. Phys. Rev. Lett. $113(9), 094501$.

PAN, Y. \& YUE, D.K.P. 2015 Decaying capillary wave turbulence under broad-scale dissipation. J. Fluid Mech. 780, R1.

PAN, Y. \& YUE, D.K.P. 2017 Understanding discrete capillary-wave turbulence using a quasi-resonant kinetic equation. J. Fluid Mech. 816, R1.

Phillips, O.M. 1958 The equilibrium range in the spectrum of wind-generated waves. J. Fluid Mech. 4 (4), 426-434.

Phillips, O.M. 1981 The dispersion of short wavelets in the presence of a dominant long wave. J. Fluid Mech. 107, 465-485.

Plant, W.J. 2003 A new interpretation of sea-surface slope probability density functions. J. Geophys. Res. 665108 (C9), 3295.

Plant, W.J., Dahl, P.H., Giovanangeli, J.-P. \& BRAnger, H. 2004 Bound and free surface waves in a large wind-wave tank. J. Geophys. Res. 109, C10002.

Plant, W.J., Keller, W.C., Hesany, V., Hara, T., Bock, E. \& Donelan, M.A. 1999 Bound waves and bragg scattering in a wind-wave tank. J. Geophys. Res. 104 (C2), 3243-3263.

Pushikarev, A.N. \& Zakharov, V.E. 1996 Turbulence of capillary waves. Phys. Rev. Lett. 76 (18), 3320.

PushkareV, A.N. \& ZAKHAROV, V.E. 2000 Turbulence of capillary waves-theory and numerical simulation. Physica D 135 (1-2), 98-116.

Stuhlmeier, R. \& Stiassnie, M. 2019 Nonlinear dispersion for ocean surface waves. J. Fluid Mech. 859, 49-58.

TANAKA, M. 2001 Verification of hasselmann's energy transfer among surface gravity waves by direct numerical simulations of primitive equations. J. Fluid Mech. 444, 199-221.

West, B.J., Brueckner, K.A., Janda, R.S., Milder, D.M. \& Milton, R.L. 1987 A new numerical method for surface hydrodynamics. J. Geophys. Res. 92 (C11), 11803-11824.

XiAO, W., LIU, Y., WU, G. \& YUE, D.K.P. 2013 Rogue wave occurrence and dynamics by direct simulations of nonlinear wave-field evolution. J. Fluid Mech. 720, 357-392.

Yokoyama, N. 2004 Statistics of gravity waves obtained by direct numerical simulation. J. Fluid Mech. 501, 169-178.

ZAKHAROV, V.E. 1968 Stability of periodic waves of finite amplitude on the surface of a deep fluid. J. Appl. Mech. Tech. Phys. 9 (2), 190-194.

ZAKHAROV, V.E. \& FilOnENKO, N.N. 1967 Energy spectrum for stochastic oscillations of the surface of a liquid. Sov. Phys. Dokl. 11, 881. 